\title{
CHARACTERIZING COMPACT FAMILIES VIA THE LAPLACE TRANSFORM
}

\author{
Mateusz Krukowski \\ Łódź University of Technology, Institute of Mathematics \\ Wólczańska 215, 90-924 Łódź, Poland; krukowski.mateusz13@gmail.com
}

\begin{abstract}
In 1985, Pego characterized compact families in $L^{2}(\mathbf{R})$ in terms of the Fourier transform. It took nearly 30 years to realize that Pego's result can be proved in a more general setting of locally compact abelian groups (works of Górka and Kostrzewa). In the current paper, we argue that the Fourier transform is not the only integral transform that is efficient in characterizing compact families and suggest the Laplace transform as a possible alternative.
\end{abstract}

\section{Introduction}

The main objective of the article is to use Laplace transform to characterize compact families in $L^{2}\left(\mathbf{R}_{+}\right)$. This task is achieved in Theorem 9, after we introduce and study the concepts of Laplace equicontinuity and equivanishing. In the author's opinion, the culminating result of the paper elegantly rhymes with the ideas of Pego and later Górka and Kostrzewa. However, before we dive into technical details, let us first lay the historical background.

Characterizing compact families has been a vital topic in function spaces' theory at least since the end of the 19th century. Around 1883, two Italian mathematicians Cesare Arzelà (1847-1912) and Giulio Ascoli (1843-1896) provided the necessary and sufficient conditions under which every sequence of a given family of real-valued continuous functions (defined on a closed and bounded interval), has a uniformly convergent subsequence (this is called sequential compactness). A couple of decades later (in 1931), Andrey Kolmogorov (1903-1987) succeeded in characterizing the compact families in $L^{p}\left(\mathbf{R}^{N}\right)$, when $1<p<\infty$ and all the functions are supported in a common bounded set (comp. [17]). A year later, Jacob David Tamarkin (1888-1945) got rid of the second restriction (comp. [25]) and in 1933, Marcel Riesz (1886-1969), a younger brother of Frigyes Riesz, proved the general case for $L^{p}\left(\mathbf{R}^{N}\right)$, where $1 \leqslant p<$ $\infty$. In 1940, a French mathematician and one of the leaders of the Bourbaki group, André Weil (1906-1998) wrote a book 'L'intégration dans les groupes topologique' (comp. [26]), in which he proved the Kolmogorov-Riesz theorem for a locally compact Hausdorff group $G$ instead of $\mathbf{R}^{N}$.

The next major contribution came over 40 years later (1985), when Robert L. Pego characterized compact families in $L^{2}(\mathbf{R})$ via the Fourier transform. This innovative idea was the cornerstone for the works of two Polish mathematicians Przemysław Górka and Tomasz Kostrzewa. In [7] and [8], they proved that a counterpart of Pego theorem holds for locally compact abelian groups (this is reminiscent of Weil's contribution). Obviously, there are other works related to the topic, which are worthmentioning: $[1,2,3,9,10,11,12,13,14,15,20,21]$ just to name a few.

https://doi.org/10.5186/aasfm.2020.4553

2010 Mathematics Subject Classification: Primary 44A10, 42A38.

Key words: Laplace transform, Pego theorem, compactness. 
In the current paper, we argue that the Fourier transform is not the only one that can be used to characterize compact families. In Section 2 we introduce the basic definitions and discuss the necessary notation. We also prove the fundamental theorems, which are very well-known in the context of the Fourier transform, and probably less known in the context of the Laplace transform. In Section 3 we prove the main results. Theorem 9, which is a counterpart of the Pego's result, is the climax of the paper.

\section{Preliminary results}

For a measurable, complex-valued function $f: \mathbf{R}_{+} \rightarrow \mathbf{C}$ and a real number $x \geqslant 0$, we denote

$$
f_{x}(t)=f(t) e^{-x t}
$$

We say that $f: \mathbf{R}_{+} \rightarrow \mathbf{C}$ is a Laplace-Pego function of order $x \geqslant 0$ if

$$
f_{x} \in L^{1}\left(\mathbf{R}_{+}\right) \cap L^{2}\left(\mathbf{R}_{+}\right) .
$$

The norms in $L^{1}\left(\mathbf{R}_{+}\right)$and $L^{2}\left(\mathbf{R}_{+}\right)$are denoted by $\|\cdot\|_{1}$ and $\|\cdot\|_{2}$, respectively. Moreover, if $\mathcal{A}$ is a subfamily of Laplace-Pego functions with a common order $x \geqslant 0$, then we denote

$$
\mathcal{A}_{x}=\left\{f_{x}: f \in \mathcal{A}\right\} .
$$

Let $f$ be a Laplace-Pego function of order $x \geqslant 0$. The Laplace transform $\mathcal{L}\{f\}$ of the function $f$ is defined by

$$
\mathcal{L}\{f\}(z)=\int_{0}^{\infty} f(t) e^{-z t} d t .
$$

A natural question arises: when does the above integral exist? To answer this question, observe that if $\operatorname{Re}(z) \geqslant x$, then

$$
\begin{aligned}
|\mathcal{L}\{f\}(z)| & =\left|\int_{0}^{\infty} f(t) e^{-\operatorname{Re}(z) t} e^{-i \operatorname{Im}(z) t} d t\right| \leqslant \int_{0}^{\infty}|f(t)| e^{-\operatorname{Re}(z) t} d t \\
& =\int_{0}^{\infty}|f(t)| e^{-x t} e^{(x-\operatorname{Re}(z)) t} d t \leqslant\left\|f_{x}\right\|_{1}<\infty .
\end{aligned}
$$

In other words, the Laplace transform $\mathcal{L}\{f\}$ exists in the half-plane $\operatorname{Re}(z) \geqslant x$.

An important special case of the Laplace transform is the Fourier transform, which we define by

$$
\widehat{f}(y)=\mathcal{L}\{f\}(2 \pi i y) .
$$

Let us formulate a crucial theorem regarding the Laplace transform, which we will use multiple times throughout the paper:

Theorem 1. (Plancherel theorem for the Laplace transform) If $f$ is a LaplacePego function of order $x \geqslant 0$, then

$$
\frac{1}{2 \pi} \int_{-\infty}^{\infty}|\mathcal{L}\{f\}(x+i y)|^{2} d y=\int_{0}^{\infty} e^{-2 x t}|f(t)|^{2} d t .
$$

Proof. At first, observe that

$$
\begin{aligned}
\forall_{y \in \mathbf{R}} \mathcal{L}\{f\}(x+i y) & =\int_{0}^{\infty} f(t) e^{-x t} e^{-i y t} d t \\
& =\int_{0}^{\infty} f(t) e^{-x t} e^{-2 \pi i \frac{y}{2 \pi} t} d t=\widehat{f}_{x}\left(\frac{y}{2 \pi}\right) .
\end{aligned}
$$


By the classical Plancherel theorem [4, Theorem 3.5.2, p. 53] or [24, Theorem 1.1, p. 208] we have

$$
\int_{-\infty}^{\infty}\left|\widehat{f}_{x}\left(\frac{y}{2 \pi}\right)\right|^{2} d \frac{y}{2 \pi}=\int_{-\infty}^{\infty}\left|f_{x}(t)\right|^{2} d t=\int_{0}^{\infty} e^{-2 x t}|f(t)|^{2} d t
$$

Upon observing that

$$
\int_{-\infty}^{\infty}\left|\widehat{f}_{x}\left(\frac{y}{2 \pi}\right)\right|^{2} d \frac{y}{2 \pi} \stackrel{(2)}{=} \frac{1}{2 \pi} \int_{-\infty}^{\infty}|\mathcal{L}\{f\}(x+i y)|^{2} d y
$$

we conclude the proof.

The theorem, which we present below, is a counterpart of a well-know result in the theory of Fourier transform:

Theorem 2. (Riemann-Lebesgue lemma for the Laplace transform) If $f$ is a Laplace-Pego function of order $x \geqslant 0$, then

$$
\lim _{y \rightarrow \pm \infty} \mathcal{L}\{f\}(x+i y)=0 .
$$

Proof. At first, let $f=\mathbb{1}_{(a, b)}$ where $(a, b) \subset \mathbf{R}_{+}$. Then, for every $y \in \mathbf{R}$ we have

$$
\begin{aligned}
\forall_{y \in \mathbf{R}} \mathcal{L}\{f\}(x+i y) & =\int_{0}^{\infty} \mathbb{1}_{(a, b)}(t) e^{-(x+i y) t} d t \\
& =\int_{a}^{b} e^{-(x+i y) t} d t=\frac{e^{-(x+i y) a}-e^{-(x+i y) b}}{x+i y},
\end{aligned}
$$

so (3) holds. By linearity of the Laplace transform, the result is also true for all simple functions.

Finally, let $f$ be an arbitrary Laplace-Pego function and let $\varepsilon>0$. Since simple functions are dense in $L^{1}\left(\mathbf{R}_{+}\right)$, there exists a simple function $g$ such that

$$
\int_{0}^{\infty}\left|f(t) e^{-x t}-g(t)\right| d t<\varepsilon
$$

Hence

$$
\begin{aligned}
\lim _{y \rightarrow \pm \infty}|\mathcal{L}\{f\}(x+i y)| & =\lim _{y \rightarrow \pm \infty}\left|\int_{0}^{\infty} f(t) e^{-x t} e^{-i y t} d t\right| \\
& \leqslant \int_{0}^{\infty}\left|f(t) e^{-x t}-g(t)\right| d t+\lim _{y \rightarrow \pm \infty}\left|\int_{0}^{\infty} g(t) e^{-i y t} d t\right| \\
& \stackrel{(4)}{<} \varepsilon+\lim _{y \rightarrow \pm \infty}|\mathcal{L}\{g\}(i y)|=\varepsilon+\lim _{y \rightarrow \pm \infty}\left|\widehat{g}\left(\frac{y}{2 \pi}\right)\right|=\varepsilon
\end{aligned}
$$

where the last equality follows from the classical Riemann-Lebesgue lemma for the Fourier transform (comp. [16, Theorem 1.7, p. 136]. Since $\varepsilon>0$ was chosen arbitrarily, we conclude the proof.

We will now recall the prominent fact that the Laplace trasnform 'changes the convolution of two functions to multiplication'. A convolution of two Laplace-Pego functions $f, g$ with a common order $x \geqslant 0$ is defined by

$$
\forall_{t>0} f \star g(t)=\int_{0}^{t} f(s) g(t-s) d s .
$$

Theorem 3. If $f, g$ are Laplace-Pego functions with a common order $x \geqslant 0$, then $(f \star g)_{x} \in L^{1}\left(\mathbf{R}_{+}\right)$. In particular, it exists almost everywhere. 
Proof. At first, let us observe that

$$
\begin{aligned}
\forall_{t>0} e^{-x t} f \star g(t) & =\int_{0}^{t} e^{-x t} f(s) g(t-s) d s \\
& =\int_{0}^{t} e^{-x s} f(s) e^{-x(t-s)} g(t-s) d s .
\end{aligned}
$$

Furthermore, by [24, Proposition 3.9, p. 86], we note that the function $F: \mathbf{R}_{+} \times$ $\mathbf{R}_{+} \longrightarrow \mathbf{C}$ defined by

$$
F(t, s)=e^{-x s} f(s) e^{-x(t-s)} g(t-s)
$$

is measurable, so we are in position to apply Tonelli's theorem. Consequently, we obtain

$$
\begin{aligned}
&\left|\int_{0}^{\infty} e^{-x t} f \star g(t) d t\right| \stackrel{(5)}{\leqslant} \int_{0}^{\infty} \int_{0}^{t} e^{-x s}|f|(s) e^{-x(t-s)}|g|(t-s) d s d t \\
& \stackrel{\text { Tonelli's thm }}{=} \int_{0}^{\infty} \int_{s}^{\infty} e^{-x s}|f|(s) e^{-x(t-s)}|g|(t-s) d t d s \\
&=\left\|f_{x}\right\|_{1}\left\|g_{x}\right\|_{1}<\infty
\end{aligned}
$$

which ends the proof.

The convolution theorem for the Laplace transform, which we present below, should be juxtaposed with [22, Theorem 2.39, p. 92].

Theorem 4. (Convolution theorem for the Laplace transform) If $f$ and $g$ are Laplace-Pego functions with a common order $x \geqslant 0$, then

$$
\mathcal{L}\{f \star g\}(z)=\mathcal{L}\{f\}(z) \cdot \mathcal{L}\{g\}(z)
$$

for $\operatorname{Re}(z) \geqslant x$.

Proof. Note that the function $F: \mathbf{R}_{+} \times \mathbf{R}_{+} \longrightarrow \mathbf{C}$ defined by

$$
F(t, s)=f(s) g(t-s) e^{-z t}
$$

is measurable, so by Tonelli's theorem we have

$$
\begin{array}{r}
\mathcal{L}\{f \star g\}(z)=\int_{0}^{\infty} f \star g(t) e^{-z t} d t=\int_{0}^{\infty} \int_{0}^{t} f(s) g(t-s) e^{-z t} d s d t \\
\stackrel{\text { Tonelli's thm }}{=} \int_{0}^{\infty} \int_{s}^{\infty} f(s) g(t-s) e^{-z t} d t d s=\mathcal{L}\{f\}(z) \cdot \mathcal{L}\{g\}(z),
\end{array}
$$

which ends the proof.

\section{Main results}

A subfamily $\mathcal{A}$ of Laplace-Pego functions with a common order $x \geqslant 0$ is said to be exponentially $L^{2}$-equivanishing at $x$, if

$$
\forall_{\varepsilon>0} \exists_{T>0} \forall_{f \in \mathcal{A}} \int_{T}^{\infty} e^{-2 x t}|f(t)|^{2} d t<\varepsilon
$$

Furthermore, we say that a family $\mathcal{A}$ is Laplace equicontinuous at $x$, if

$$
\forall_{\varepsilon>0} \exists_{\delta>0} \forall_{f \in \mathcal{A}} \frac{1}{2 \pi} \int_{-\infty}^{\infty}|\mathcal{L}\{f\}(x+i y+\delta)-\mathcal{L}\{f\}(x+i y)|^{2} d y<\varepsilon .
$$

We will now relate the concepts of Laplace equicontinuity and exponential $L^{2}$ equivanishing. 
Theorem 5. Let $\mathcal{A}$ be a subfamily of Laplace-Pego functions with a common order $x \geqslant 0$. If $\mathcal{A}$ is Laplace equicontinuous at $x$, then it is exponentially $L^{2}$ equivanishing at $x$. Furthermore, if $\mathcal{A}_{x}$ is $L^{2}$-bounded, then the implication can be reversed.

Proof. We divide the proof into two steps:

Step 1. At first, we assume that $\mathcal{A}$ is Laplace equicontinuous at $x$, so for a fixed $\varepsilon>0$ we may choose $\delta>0$ according to (7). Let $T>0$ be such that

$$
\left|e^{-\delta T}-1\right|^{2} \geqslant \frac{1}{2}
$$

Consequently, for every $f \in \mathcal{A}$ we obtain

$$
\begin{aligned}
& \varepsilon>\frac{1}{2 \pi} \int_{-\infty}^{\infty}|\mathcal{L}\{f\}(x+i y+\delta)-\mathcal{L}\{f\}(x+i y)|^{2} d y \\
& \quad=\frac{1}{2 \pi} \int_{-\infty}^{\infty}\left|\int_{0}^{\infty} f(t)\left(e^{-\delta t}-1\right) e^{-(x+i y) t} d t\right|^{2} d y \\
& \stackrel{\text { Theorem } 1}{=} \int_{0}^{T} e^{-2 x t}|f(t)|^{2}\left|e^{-\delta t}-1\right|^{2} d t+\int_{T}^{\infty} e^{-2 x t}|f(t)|^{2}\left|e^{-\delta t}-1\right|^{2} d t \\
& \quad \stackrel{(8)}{\geqslant} \frac{1}{2} \int_{T}^{\infty} e^{-2 x t}|f(t)|^{2} d t,
\end{aligned}
$$

which ends the first part of the proof.

Step 2. At this point, we assume that $\mathcal{A}_{x}$ is $L^{2}$-bounded, so there exists $M>0$ such that

$$
\forall_{f \in \mathcal{A}} \int_{0}^{\infty} e^{-2 x t}|f(t)|^{2} d t \leqslant M
$$

We will show that if $\mathcal{A}$ is exponentially $L^{2}$-equivanishing at $x$, then it is Laplace equicontinuous at $x$.

Fix $\varepsilon>0$ and choose $T>0$ as in the definition of the exponential $L^{2}$-equivanishing (6). Let $\delta>0$ be such that

$$
\left|e^{-\delta T}-1\right|^{2} M<\varepsilon
$$

We have

$$
\begin{aligned}
& \frac{1}{2 \pi} \int_{-\infty}^{\infty}|\mathcal{L}\{f\}(x+i y+\delta)-\mathcal{L}\{f\}(x+i y)|^{2} d y \\
& \stackrel{\text { Theorem } 1}{=} \int_{0}^{T} e^{-2 x t}|f(t)|^{2}\left|e^{-\delta t}-1\right|^{2} d t+\int_{T}^{\infty} e^{-2 x t}|f(t)|^{2}\left|e^{-\delta t}-1\right|^{2} d t \\
& \quad \stackrel{(9)}{\leqslant}\left|e^{-\delta T}-1\right|^{2} M+\int_{T}^{\infty} e^{-2 x t}|f(t)|^{2} d t \stackrel{(6)}{<} 2 \varepsilon,
\end{aligned}
$$

which ends the proof.

A subfamily $\mathcal{A}$ of Laplace-Pego functions with a common order $x \geqslant 0$ is said to be exponentially $L^{2}$-equicontinuous at $x$, if

$$
\forall_{\varepsilon>0} \exists_{\delta>0} \forall_{\substack{s \in(0, \delta) \\ f \in \mathcal{A}}}\left(\int_{0}^{\infty} e^{-2 x t}|f(t)-f(t-s)|^{2} d t\right)^{\frac{1}{2}}<\varepsilon .
$$

Let us make one technical remark at this point. Although we defined the domain of the Laplace-Pego functions as $\mathbf{R}_{+}$, we may actually treat these functions as functions 
on $\mathbf{R}$ with the property $f(t)=0$ if $t<0$. Consequently, in (10) we have $f(t-s)=0$ whenever $s>t$.

Furthermore, we say that a family $\mathcal{A}$ is Laplace equivanishing at $x$, if

$$
\forall_{\varepsilon>0} \exists_{T>0} \forall_{f \in \mathcal{A}} \int_{\mathbf{R} \backslash[-T, T]}|\mathcal{L}\{f\}(x+i y)|^{2} d y<\varepsilon .
$$

We study the relationship between the novel notion of the exponential $L^{2}$-equicontinuity of $\mathcal{A}$ and the classical equicontinuity of $\mathcal{A}_{x}$ in the lemma below:

Lemma 6. Let $\mathcal{A}$ be a subfamily of Laplace-Pego functions with a common order $x \geqslant 0$. If $\mathcal{A}_{x}$ is $L^{2}$-bounded then $\mathcal{A}$ is exponentially $L^{2}$-equicontinuous at $x$ if and only if $\mathcal{A}_{x}$ is $L^{2}$-equicontinuous, i.e.

$$
\forall_{\varepsilon>0} \exists_{\delta>0} \forall_{\substack{s \in(0, \delta), f \in \mathcal{A}}}^{\infty}\left|e_{0}^{-x(t+s)} f(t+s)-e^{-x t} f(t)\right|^{2} d t<\varepsilon .
$$

Proof. Since $\mathcal{A}_{x}$ is $L^{2}$-bounded, there exists $M>0$ such that

$$
\forall f \in \mathcal{A}\left(\int_{0}^{\infty} e^{-2 x t}|f(t)|^{2} d t\right)^{\frac{1}{2}} \leqslant M .
$$

We divide the proof of the lemma into two steps:

Step 1. In the first part of the proof, we assume that the family $\mathcal{A}$ is exponentially $L^{2}$-equicontinuous at $x$. We fix $\varepsilon>0$ and choose $\delta>0$ such that

- (12) is satisfied, and

- for every $s \in(0, \delta)$ we have

$$
\int_{0}^{s} e^{-2 x t}|f(t)|^{2} d t<\varepsilon
$$

which is possible due to Theorem 8 in [18], p. 148, and

- for every $s \in(0, \delta)$ we have

$$
\left|e^{-x s}-1\right| M<\varepsilon \text {. }
$$

Consequently, for every $s \in(0, \delta)$ and $f \in \mathcal{A}$ we have

$$
\begin{aligned}
& \left(\int_{0}^{\infty} e^{-2 x t}|f(t)-f(t-s)|^{2} d t\right)^{\frac{1}{2}}=\left(\int_{-s}^{\infty} e^{-2 x(t+s)}|f(t+s)-f(t)|^{2} d t\right)^{\frac{1}{2}} \\
& \leqslant\left(\int_{-s}^{\infty} e^{-2 x(t+s)}\left|f(t+s)-e^{x s} f(t)\right|^{2} d t\right)^{\frac{1}{2}}+\left(\int_{-s}^{\infty} e^{-2 x(t+s)}\left|e^{x s} f(t)-f(t)\right|^{2} d t\right)^{\frac{1}{2}} \\
& =\left(\int_{-s}^{0} e^{-2 x(t+s)}|f(t+s)|^{2} d t+\int_{0}^{\infty} e^{-2 x(t+s)}\left|f(t+s)-e^{x s} f(t)\right|^{2} d t\right)^{\frac{1}{2}} \\
& +\left(\int_{0}^{\infty} e^{-2 x(t+s)}|f(t)|^{2}\left|e^{x s}-1\right|^{2} d t\right)^{\frac{1}{2}} \\
& \leqslant\left(\int_{0}^{s} e^{-2 x t}|f(t)|^{2} d t+\int_{0}^{\infty}\left|e^{-x(t+s)} f(t+s)-e^{-x t} f(t)\right|^{2} d t\right)^{\frac{1}{2}} \\
& \quad+\left|e^{-x s}-1\right| M \stackrel{(12),(13),(14)}{\leqslant}(2 \varepsilon)^{\frac{1}{2}}+\varepsilon .
\end{aligned}
$$

Since $\varepsilon>0$ was chosen arbitrarily, the above estimates end the first part of the proof. 
Step 2. In this part of the proof, we assume that $\mathcal{A}_{x}$ is $L^{2}$-equicontinuous. Again, we fix $\varepsilon>0$ and let $\delta>0$ be such that

- (10) is satisfied, and

- for every $s \in(0, \delta)$ we have

$$
\left|1-e^{x s}\right| M<\varepsilon \quad \text { and } \quad e^{x s} \leqslant 2 .
$$

For every $s \in(0, \delta)$ and $f \in \mathcal{A}$ we have

$$
\begin{aligned}
& \left(\int_{0}^{\infty}\left|e^{-x(t+s)} f(t+s)-e^{-x t} f(t)\right|^{2} d t\right)^{\frac{1}{2}}=\left(\int_{s}^{\infty}\left|e^{-x t} f(t)-e^{-x(t-s)} f(t-s)\right|^{2} d t\right)^{\frac{1}{2}} \\
& \leqslant\left(\int_{s}^{\infty}\left|e^{-x t} f(t)-e^{-x(t-s)} f(t)\right|^{2} d t\right)^{\frac{1}{2}}+\left(\int_{s}^{\infty}\left|e^{-x(t-s)} f(t)-e^{-x(t-s)} f(t-s)\right|^{2} d t\right)^{\frac{1}{2}} \\
& \leqslant\left|1-e^{x s}\right|\left(\int_{0}^{\infty} e^{-2 x t}|f(t)|^{2} d t\right)^{\frac{1}{2}}+e^{x s} \varepsilon \stackrel{(15)}{\leqslant}\left|1-e^{x s}\right| M+2 \varepsilon<3 \varepsilon,
\end{aligned}
$$

which ends the proof.

We will now study the relationship between the exponential $L^{2}$-equicontinuity and the Laplace equivanishing.

Theorem 7. Let $\mathcal{A}$ be a subfamily of Laplace-Pego functions with a common order $x \geqslant 0$. Exponential $L^{2}$-equicontinuity at $x$ implies Laplace equivanishing at $x$. Furthermore, if $\mathcal{A}_{x}$ is $L^{2}$-bounded, then the implication can be reversed.

Proof. We divide the proof into two steps:

Step 1 . We assume that the family $\mathcal{A}$ is exponentially $L^{2}$-equicontinuous at $x$, so for a fixed $\varepsilon>0$ we can choose $\delta>0$ according to the exponential $L^{2}$ equicontinuity (10). Let $g$ be a nonnegative and continuous function on $\mathbf{R}_{+}$such that $\operatorname{supp}(g) \subset(0, \delta)$ and $\int_{0}^{\infty} g(s) d s=1$. Naturally, $g$ is a Laplace-Pego function of order $x$.

By Theorem 2, let $T>0$ be such that

$$
\forall_{y \in \mathbf{R} \backslash[-T, T]}|\mathcal{L}\{g\}(x+i y)| \leqslant \frac{1}{2} .
$$

Consequently, we have

$$
\begin{aligned}
\forall_{f \in \mathcal{A}} & \left(\int_{\mathbf{R} \backslash[-T, T]}|\mathcal{L}\{f\}(x+i y)|^{2} d y\right)^{\frac{1}{2}} \\
\leqslant & \left(\int_{\mathbf{R} \backslash[-T, T]}|\mathcal{L}\{f\}(x+i y)(1-\mathcal{L}\{g\}(x+i y))|^{2} d y\right)^{\frac{1}{2}} \\
& +\left(\int_{\mathbf{R} \backslash[-T, T]}|\mathcal{L}\{f\}(x+i y) \mathcal{L}\{g\}(x+i y)|^{2} d y\right)^{\frac{1}{2}} \\
\stackrel{(16)}{\leqslant} & \left(\int_{\mathbf{R} \backslash[-T, T]}|\mathcal{L}\{f\}(x+i y)(1-\mathcal{L}\{g\}(x+i y))|^{2} d y\right)^{\frac{1}{2}} \\
& +\frac{1}{2}\left(\int_{\mathbf{R} \backslash[-T, T]}|\mathcal{L}\{f\}(x+i y)|^{2} d y\right)^{\frac{1}{2}},
\end{aligned}
$$


which implies

$$
\begin{aligned}
& \forall_{f \in \mathcal{A}}\left(\int_{\mathbf{R} \backslash[-T, T]}|\mathcal{L}\{f\}(x+i y)|^{2} d y\right)^{\frac{1}{2}} \\
& \leqslant 2\left(\int_{\mathbf{R} \backslash[-T, T]}|\mathcal{L}\{f\}(x+i y)(1-\mathcal{L}\{g\}(x+i y))|^{2} d y\right)^{\frac{1}{2}} \\
& \stackrel{\text { Theorem }}{\leqslant} 2\left(\int_{\mathbf{R}}|\mathcal{L}\{f\}(x+i y)-\mathcal{L}\{f \star g\}(x+i y)|^{2} d y\right)^{\frac{1}{2}} \\
& \stackrel{\text { Theorem } 1}{=} 2 \sqrt{2 \pi}\left(\int_{0}^{\infty} e^{-2 x t}|f(t)-f \star g(t)|^{2} d t\right)^{\frac{1}{2}} \\
& =2 \sqrt{2 \pi}\left(\int_{0}^{\infty} e^{-2 x t}\left|f(t)-\int_{0}^{\infty} f(t-s) g(s) d s\right|^{2} d t\right)^{\frac{1}{2}} \\
& =2 \sqrt{2 \pi}\left(\int_{0}^{\infty}\left|\int_{0}^{\infty} e^{-x t}(f(t)-f(t-s)) g(s) d s\right|^{2} d t\right)^{\frac{1}{2}} \\
& \text { Minkowski integral } \\
& \stackrel{\substack{\text { knewski integral } \\
\leqslant}}{\leqslant} 2 \sqrt{2 \pi} \int_{0}^{\infty}\left(\int_{0}^{\infty} e^{-2 x t}|f(t)-f(t-s)|^{2}|g(s)|^{2} d t\right)^{\frac{1}{2}} d s \\
& =2 \sqrt{2 \pi} \int_{0}^{\infty} g(s)\left(\int_{0}^{\infty} e^{-2 x t}|f(t)-f(t-s)|^{2} d t\right)^{\frac{1}{2}} d s \\
& =2 \sqrt{2 \pi} \int_{0}^{\delta} g(s)\left(\int_{0}^{\infty} e^{-2 x t}|f(t)-f(t-s)|^{2} d t\right)^{\frac{1}{2}} d s \\
& \leqslant 2 \sqrt{2 \pi} \varepsilon \int_{0}^{\delta} g(s) d s=2 \sqrt{2 \pi} \varepsilon .
\end{aligned}
$$

Let us remark that the use of Minkowski inequality in the above estimates is justified, because the function $F(t, s)=e^{-2 x t}|f(t)-f(t-s)|^{2}|g(s)|^{2}$ is measurable due to [24, Proposition 3.9, p. 86]. Since $\varepsilon>0$ was chosen arbitrarily, the above estimates end the first part of the proof.

Step 2. For this part of the proof, we assume that $\mathcal{A}_{x}$ is $L^{2}$-bounded, so there exists $M_{1}>0$ such that

$$
\forall_{f \in \mathcal{A}} \int_{0}^{\infty} e^{-2 x t}|f(t)|^{2} d t \leqslant M_{1}
$$

We will show that if $\mathcal{A}$ is Laplace equivanishing at $x$ then it is exponentially $L^{2}$ equicontinuous at $x$. For convenience, we denote $T_{-s} f(t)=f(t-s)$. We observe the following equalities

$$
\begin{aligned}
& \underset{\substack{s>0 \\
f \in \mathcal{A}}}{\forall} \int_{0}^{\infty} e^{-2 x t}|f(t)-f(t-s)|^{2} d t=\int_{0}^{\infty} e^{-2 x t}\left|f(t)-T_{-s} f(t)\right|^{2} d t \\
& \stackrel{\text { Theorem } 1}{=} \frac{1}{2 \pi} \int_{-\infty}^{\infty}\left|\mathcal{L}\left\{f-T_{-s} f\right\}(x+i y)\right|^{2} d y \\
& =\frac{1}{2 \pi} \int_{-\infty}^{\infty}\left|\mathcal{L}\{f\}(x+i y)-e^{-s(x+i y)} \mathcal{L}\{f\}(x+i y)\right|^{2} d y
\end{aligned}
$$




$$
=\frac{1}{2 \pi} \int_{-\infty}^{\infty}\left|1-e^{-s(x+i y)}\right|^{2}|\mathcal{L}\{f\}(x+i y)|^{2} d y .
$$

Fix $\varepsilon>0$ and choose $T>0$ according to Laplace equivanishing (11). Let $\delta>0$ be such that

$$
\underset{\substack{s \in(0, \delta) \\ y \in[-T, T]}}{\forall}\left|1-e^{-s(x+i y)}\right|^{2}<\varepsilon,
$$

and put

$$
M_{2}=\max _{\substack{s \in[0, \delta], y \in \mathbf{R}}}\left|1-e^{-s(x+i y)}\right|^{2},
$$

which is finite due to

$$
M_{2} \leqslant \max _{s \in[0, \delta]}\left(1+e^{-s x}\right)^{2} .
$$

Finally, for every $s \in(0, \delta)$ and $f \in \mathcal{A}$, we have

$$
\begin{aligned}
& \int_{0}^{\infty} e^{-2 x t}|f(t)-f(t-s)|^{2} d t \stackrel{(18)}{=} \frac{1}{2 \pi} \int_{-T}^{T}\left|1-e^{-s(x+i y)}\right|^{2}|\mathcal{L}\{f\}(x+i y)|^{2} d y \\
& +\frac{1}{2 \pi} \int_{\mathbf{R} \backslash[-T, T]}\left|1-e^{-s(x+i y)}\right|^{2}|\mathcal{L}\{f\}(x+i y)|^{2} d y \\
& \stackrel{(19),(20)}{\leqslant} \frac{\varepsilon}{2 \pi} \int_{-T}^{T}|\mathcal{L}\{f\}(x+i y)|^{2} d y+\frac{M_{2}}{2 \pi} \int_{\mathbf{R} \backslash[-T, T]}|\mathcal{L}\{f\}(x+i y)|^{2} d y \\
& \stackrel{\text { Theorem 1, (11) }}{\leqslant} \int_{0}^{\infty} e^{-2 x t}|f(t)|^{2} d t+\frac{M_{2}}{2 \pi} \varepsilon \leqslant\left(M_{1}+\frac{M_{2}}{2 \pi}\right) \varepsilon .
\end{aligned}
$$

Since $\varepsilon>0$ was chosen arbitrarily, we conclude the proof.

Before we present the final theorem of the paper, let us recall the celebrated Riesz-Kolmogorov theorem:

Theorem 8. (Riesz-Kolmogorov theorem, comp. [14]) $A$ family $\mathcal{A} \subset L^{2}\left(\mathbf{R}_{+}\right)$is relatively compact if and only if

- $\mathcal{A}$ is $L^{2}$-bounded;

- $\mathcal{A}$ is $L^{2}$-equicontinuous;

- $\mathcal{A}$ is $L^{2}$-equivanishing.

The final theorem, which is the climax of the paper, should be juxtaposed with Pego theorem in [7], [8] and [19].

Theorem 9. Let $\mathcal{A}$ be a subfamily of Laplace-Pego functions with a common order $x$ and such that $\mathcal{A}_{x}$ is $L^{2}$-bounded. The family $\mathcal{A}_{x}$ is relatively compact in $L^{2}\left(\mathbf{R}_{+}\right)$if and only if

- $\mathcal{A}$ is Laplace equicontinuous at $x$

- $\mathcal{A}$ is Laplace equivanishing at $x$.

Proof. The proof is divided into two steps:

Step 1. We assume that $\mathcal{A}$ is Laplace equicontinuous and equivanishing at $x$. At first, we note that Laplace equicontinuity of $\mathcal{A}$ at $x$ implies that this family is exponentially $L^{2}$-equivanishing at $x$ (Theorem 5). In other words, $\mathcal{A}_{x}$ is $L^{2}$-equivanishing. 
Furthermore, Laplace equivanishing of $\mathcal{A}$ at $x$ implies that this family is exponentially $L^{2}$-equicontinuous (Theorem 7). In other words, $\mathcal{A}_{x}$ is $L^{2}$-equicontinuous (Lemma 6). By Theorem 8, we conclude that $\mathcal{A}_{x}$ is relatively compact in $L^{2}\left(\mathbf{R}_{+}\right)$.

Step 2. For the second part of the proof, we assume that $\mathcal{A}_{x}$ is relatively compact in $L^{2}\left(\mathbf{R}_{+}\right)$. By Theorem 8, the family $\mathcal{A}_{x}$ is $L^{2}$-equicontinuous and $L^{2}$-equivanishing. $L^{2}$-equicontinuity of $\mathcal{A}_{x}$ implies that $\mathcal{A}$ is Laplace equivanishing at $x$ (Lemma 6 and Theorem 7). Moreover, $L^{2}$-equivanishing of $\mathcal{A}_{x}$ implies that $\mathcal{A}$ is Laplace equicontinuous at $x$ (Theorem 5 ), which ends the proof.

Let us conclude the paper with a simple example exhibiting how Theorem 9 works: let $\beta \in \mathbf{R}$ and let $\mathcal{A}$ be the family of functions of the form $e^{\alpha t} \cdot \mathbb{1}_{[0, \infty)}(t)$, where $\alpha \leqslant \beta$. Every $x>\beta$ is a common order for the family $\mathcal{A}$. However, it is easy to see that the family is not of order $\beta$.

Let us fix $x>\beta$. We remark that

$$
\underset{\operatorname{Re}(z) \geqslant x}{\forall \in \mathcal{A}} \mathcal{L}\{f\}(z)=\int_{0}^{\infty} e^{(\alpha-z) t} d t=\frac{-1}{\alpha-z} .
$$

Furthermore, we observe that

$$
\mathcal{A}_{x}=\left\{e^{(\alpha-x) t} \cdot \mathbb{1}_{[0, \infty)}(t): \alpha \leqslant \beta\right\}
$$

is $L^{2}$-bounded. In order to apply Theorem 9 we first check that $\mathcal{A}$ is Laplace equicontinuous at $x$. For a fixed $\varepsilon>0$ we choose $\delta>0$ such that

$$
\frac{\delta^{2}}{2(\beta-x-\delta)^{2} \cdot|\beta-x|}<\varepsilon
$$

Consequently, for every $f \in \mathcal{A}$ (or every $\alpha \leqslant \beta$ equivalently) we have

$$
\begin{aligned}
& \frac{1}{2 \pi} \int_{-\infty}^{\infty}|\mathcal{L}\{f\}(x+i y+\delta)-\mathcal{L}\{f\}(x+i y)|^{2} d y \\
& =\frac{1}{2 \pi} \int_{-\infty}^{\infty}\left|\frac{-1}{\alpha-(x+i y+\delta)}+\frac{1}{\alpha-(x+i y)}\right|^{2} d y \\
& =\frac{\delta^{2}}{2 \pi} \int_{-\infty}^{\infty} \frac{d y}{|\alpha-(x+i y+\delta)|^{2} \cdot|\alpha-(x+i y)|^{2}} \\
& =\frac{\delta^{2}}{2 \pi} \int_{\infty}^{-\infty} \frac{d y}{\left((\alpha-x-\delta)^{2}+y^{2}\right) \cdot\left((\alpha-x)^{2}+y^{2}\right)} \\
& \leqslant \frac{\delta^{2}}{2 \pi(\alpha-x-\delta)^{2}} \int_{-\infty}^{\infty} \frac{d y}{(\alpha-x)^{2}+y^{2}} \\
& =\frac{\delta^{2}}{2(\alpha-x-\delta)^{2} \cdot|\alpha-x|} \leqslant \frac{\delta^{2}}{2(\beta-x-\delta)^{2} \cdot|\beta-x|} \stackrel{(22)}{<} \varepsilon
\end{aligned}
$$

where we used the formula

$$
\forall_{b>0} \int \frac{d y}{y^{2}+b}=\frac{1}{\sqrt{b}} \arctan \left(\frac{y}{\sqrt{b}}\right)+\text { const. }
$$

Since $\varepsilon>0$ was chosen arbitrarily, we conclude that $\mathcal{A}$ is Laplace equicontinuous at $x$. 
To prove that $\mathcal{A}$ is Laplace equivanishing it suffices to pick $T>\frac{2}{\varepsilon}$ and observe that

$$
\begin{aligned}
\int_{\mathbf{R} \backslash[-T, T]}|\mathcal{L}\{f\}(x+i y)|^{2} d y & =\int_{\mathbf{R} \backslash[-T, T]} \frac{d y}{|\alpha-(x+i y)|^{2}} \\
& \leqslant \int_{\mathbf{R} \backslash[-T, T]} \frac{d y}{y^{2}}=2 \int_{T}^{\infty} \frac{d y}{y^{2}}=\frac{2}{T}<\varepsilon .
\end{aligned}
$$

To sum up, we have verified that $\mathcal{A}$ is Laplace equicontinuous and Laplace equivanishing. By Theorem 9 we conclude that $(21)$ is relatively compact in $L^{2}$.

Acknowledgements. I would like to express my deep gratitude towards Przemysław Górka for the careful study of my paper. He provided invaluable comments and remarks, which improved the article tremendously. Furthermore, I wish I could find the right words of appreciation for the anonymous Reviewer. He or she has painstakingly criticized my paper pointing out various incongruencies in the text. Without such an assistance, the paper could not have reached its final form. I feel honoured to have had my article reviewed by such a supportive and proficient person.

\section{References}

[1] Bandaliyev, R. A.: Compactness criteria in weighted variable Lebesgue spaces. - Miskolc Math. Notes 18:1, 2017, 95-101.

[2] Bandaliyev, R., and P. Górka: Relatively compact sets in variable-exponent Lebesgue spaces. - Banach J. Math. Anal. 12:2, 2018, 331-346.

[3] Bandaliyev, R. A., and S. G. Hasanov: On denseness of $C_{0}^{\infty}(\Omega)$ and compactness in $L^{p(x)}$ for $0<p(x)<1$. - Mosc. Math. J. 1:18, 2018, 1-13.

[4] Deitmar, A.: A first course in harmonic analysis. - Springer-Verlag, New York, 2005.

[5] Deitmar, A., and S. Echterhoff: Principles of harmonic analysis. - Springer, New York, 2009.

[6] Folland, G. B.: Real analysis: Modern techniques and their applications. - John Wiley and Sons, New York, 1999.

[7] Górka, P.: Pego theorem on locally compact abelian groups. - J. Algebra Appl. 14:4, Article ID $1350143,2014$.

[8] Górka, P., and T. Kostrzewa: Pego everywhere. - J. Algebra Appl. 15:4, Article ID 1650074, 2016.

[9] Górka, P., and A. Macios: The Riesz-Kolmogorov theorem on metric spaces. - Miskolc Math. Notes 15:2, 2014, 459-465.

[10] Górka, P., and A. Macios: Almost everything you need to know about relatively compact sets in variable Lebesgue spaces. - J. Funct. Anal. 269:7, 2015, 1925-1949.

[11] Górka, P., and P. Pośpiech: Banach function spaces on locally compact groups. - Annales of Functional Analysis (to appear).

[12] Górka P., and H. Rafeiro: From Arzelà-Ascoli to Riesz-Kolmogorov. - Nonlinear Anal. 144, 2016, 23-31.

[13] Górka P., and H. Rafeiro: Light side of compactness in Lebesgue spaces: Sudakov theorem. - Ann. Acad. Sci. Fenn. Math. 42, 2017, 135-139.

[14] Hanche-Olsen, H., and H. Holden: The Kolmogorov-Riesz compactness theorem. - Expo. Math. 28:4, 2010, 385-394.

[15] Hanche-Olsen, H., H. Holden, and E. Malinnikova: An improvement of the KolmogorovRiesz compactness theorem. - Expo. Math., 2018. 
[16] Katznelson, Y.: An introduction to harmonic analysis. - Cambridge Univ. Press, Cambridge, 2004.

[17] Kolmogorov, A. N.: Über Kompaktheit der Funktionenmengen bei der Konvergenz im Mittel. - Nachr. Akad. Wiss. Göttingen 9, 1931, 60-63.

[18] Natanson, I. P.: Theory of functions of a real variable. - Dover Publications, New York, 2016.

[19] Pego, R. L.: Compactness in $L^{2}$ and the Fourier transform. - Proc. Amer. Math. Soc. 95:2, 1985, 252-254.

[20] Rafeiro, H.: Kolmogorov compactness criterion in variable exponent Lebesgue spaces. - Proc. A. Razmadze Math. Inst. 150, 2009, 105-113.

[21] Rafeiro, H., and A. Vargas: On the compactness in grand spaces. - Georgian Math. J. 22:1, 2015, 141-152.

[22] Schiff, J. L.: The Laplace transform. Theory and applications. - Springer-Verlag, New York, 1999.

[23] Stein, E.: Singular integrals and differentiability properties of functions. - Princeton Univ. Press, Princeton, 1970.

[24] Stein, E., and R. Shakarchi: Real analysis: measure theory, integration and Hilbert spaces. - Princeton Univ. Press, Princeton, 2005.

[25] Tamarkin, J. D.: On the compactness of the space $L^{p}$. - Bull. Amer. Math. Soc. 32, 1932, 79-84.

[26] WeIL, A.: L'intégration dans les groupes topologiques et ses applications. - Hermann, Paris, 1965.

Received 9 June 2019 • Accepted 13 December 2019 